\title{
DÉBAT HOLLANDE-SARKOZY (2012) : ÉTUDE SÉMIOTIQUE D'UN RÉSEAU AXIOLOGIQUE ${ }^{1}$
}

\begin{abstract}
Amir Biglari $^{2}$
Cet article propose une analyse sémiotique du débat de l'entredeux-tours de l'élection présidentielle française en 2012, entre François Hollande et Nicolas Sarkozy. Il est centré sur le rassemblement, qui est une valeur essentielle à partir de laquelle la structure narrative se construit, et autour de laquelle d'autres valeurs se mettent en place : la justice, l'espoir, la confiance, le respect, la compréhension, la proximité... On met l'accent sur la tentative de chacun des candidats d'en faire des valeurs exclusives, ce qui devient un leitmotiv conflictuel tout au long du débat.
\end{abstract}

Le débat peut être considéré comme un moment privilégié de l'apparition des systèmes axiologiques des deux partenaires de l'énonciation. Le cas du débat politique, puisqu'il se déroule sur la scène publique, est particulièrement intéressant. L'un des débats politiques les plus suivis actuellement en France, c'est celui de l'entre-deux-tours de l'élection présidentielle. Il s'agit de la fin d'une longue course, non pas

1 Une première version de cet article, orientée davantage vers la problématique de la valeur en théorie sémiotique, a été publiée : (2015) Rassemblement comme valeur, dans A. Biglari (Dir.), Valeurs : aux fondements de la sémiotique (pp. 557-578), Paris : L'Harmattan.

2 Amir Biglari est Chercheur associé au Centre de recherches sémiotiques de l’Université de Limoges.

Recherches en communication, $\mathrm{n}^{\circ} 41$ (2014). 
entre plusieurs candidats comme au premier tour, mais uniquement entre deux candidats. Ces quelques heures de débat constituent un moment d'intensité maximale, c'est en général le moment le plus concentré et le plus tonique de la campagne électorale. Les deux " héros » se confrontent. Outre les idées et les thèmes présentés par chaque candidat, il y a des stratégies mises en œuvre, des modes d'énonciation engagés, des passions manifestées, des valeurs impliquées...

Cette étude portera sur le débat de l'entre-deux-tours de l'élection présidentielle française entre François Hollande et Nicolas Sarkozy, qui a eu lieu le 12 juin 2012. En faisant appel à la théorie sémiotique, nous nous proposons d'analyser un réseau axiologique qui apparaît dans ce débat, un réseau qui a pour noyau le rassemblement et auquel s'articulent d'autres valeurs telles que la justice, la confiance, l'espoir, le respect, la proximité, etc. Compte tenu du rôle prépondérant qu'y joue le rassemblement - qui s'oppose à l'antivaleur de la division -, avant de nous pencher sur ce discours, il nous a semblé utile de situer brièvement la question du rassemblement dans une perspective plus large, au niveau de l'« impersonnel de l'énonciation »" c'est-à-dire en amont de sa prise en charge par des sujets d'énonciation. Pour cela, nous partirons des définitions du dictionnaire, en nous basant sur quatre termes suggérés par les énoncés du corpus politique, deux synonymes et deux antonymes : rassembler et réunir $v s$ diviser et opposer ${ }^{2}$.

1 En se fondant sur les travaux de Hjelmslev et de Greimas, notamment sur la notion d' « usage », Denis Bertrand explique que toute énonciation individuelle est surdéterminée par un aspect impersonnel, déjà existant, de l'énonciation, car elle ne peut pas « être envisagée indépendamment de l'immense corps des énonciations collectives qui l'ont précédée et qui la rendent possible. La sédimentation des structures signifiantes, résultant de l'histoire, détermine tout acte de langage. Il y a du sens «déjà là», déposé dans la mémoire culturelle, archivé dans la langue et les significations lexicales, fixé dans les schèmes discursifs, contrôlé par les codifications des genres et des formes d'expression que l'énonciateur, lors de l'exercice individuel de la parole, convoque, actualise, réitère, ressasse, ou au contraire révoque, récuse, renouvelle et transforme ». Bertrand, D. (2000). Précis de sémiotique littéraire (pp. 55-56). Paris : Nathan.

2 Ce qui nous intéresse ici, ce ne sont pas des lexèmes en tant que tels, mais des sémèmes, c'est-à-dire des configurations construites par l'analyse (par exemple, un discours pourrait contenir l'idée du rassemblement sans que le terme ou ses synonymes ne soient utilisés). En réalité, nous considérons les lexèmes, à la suite de Greimas, comme des condensations de brefs discours, qui comprennent des structures narratives et discursives sous-jacentes. Celles-ci peuvent devenir accessibles à partir des définitions des lexèmes, qui en sont des expansions (cf. par exemple : Greimas, A.J. (1986). De la nostalgie, Étude de sémantique lexicale. Actes sémiotiques-bulletin, 39, pp. 5-11. Paris : EHESS-CNRS. Dans A. Hénault (Dir.). (2002). Questions de sémiotique (pp. 593-600). Paris : PUF.) 


\section{Définitions du dictionnaire}

Le Petit Robert définit les verbes "diviser 》 et " opposer » respectivement comme : "Semer la discorde, la désunion entre (des personnes, des groupes) »; « Mettre en face, face à face pour le combat». D'autre part, les verbes « rassembler » et « réunir » sont respectivement ainsi définis : " Assembler de nouveau (des personnes séparées). Faire venir au même endroit (des personnes) »; «Unir de nouveau, rassembler, réconcilier », « Mettre ensemble, faire communiquer $»^{1}$.

Il s'agit évidemment de deux mouvements contraires, l'un relevant d'une structure polémique ; l'autre, d'une structure participative (conflit $v s$ accord). Ces deux structures correspondent à des univers axiologiques distincts si l'on adopte l'hypothèse de la sémiotique tensive ${ }^{2}$.

Rappelons que, selon cette hypothèse, chaque valeur est définie comme le produit de l'intersection de deux valences orientées et corrélées : une valence extensive qui contrôle les tensions et pressions entre /concentré/ et /diffus/, et une valence intensive qui commande les tensions et pressions entre /tonique/ et /atone/. Aussi les deux cas extrêmes seront-ils : (i) valeur d'une intensité maximale et d'une extensité minimale ; (ii) valeur d'une intensité minimale et d'une extensité maximale. Conformément à la terminologie adoptée par Claude Zilberberg, ces deux positions correspondent respectivement à la « valeur d'absolu » et à la « valeur d'univers ».

Dans cette optique, la division et l'opposition sont de l'ordre de la valeur d'absolu, alors que le rassemblement et la réunion sont de l'ordre de la valeur d'univers : le premier mouvement, du point de vue de la « syntaxe extensive », correspond à l'opération du « tri », à savoir à la mise à l'écart et à l'exclusion, ce qui implique l'augmentation de l'intensité et de la tension ; alors que le deuxième mouvement

1 Nous nous sommes contenté des définitions qui nous ont paru plus pertinentes dans le cadre de ce travail.

2 Cf. Fontanille, J., \& Zilberberg, C. (1998). Tension et signification. Liège : Mardaga ; Zilberberg, C. (1999). Sémiotique de la douceur. Tópicos del Seminario, Benemérita Universidad Autónoma de Puebla, 2, 31-64. Disponible sur : http://www. claudezilberberg.net/download/downset.htm ; Zilberberg, C. (2001). De l'affect à la valeur. Dans M. Castellana (Dir.), Texte et valeur (pp. 43-78). Paris : L'Harmattan ; Zilberberg, C. (2006). Éléments de grammaire tensive. Limoges : PULIM; Zilberberg, C. (2008). Pour saluer l'événement. Nouveaux Actes Sémiotiques. Disponible sur : http://revues.unilim.fr/nas/document.php?id=2485 ; Zilberberg, C. (2011). Des formes de vie aux valeurs. Paris : PUF ; Zilberberg, C. (2012). La Structure tensive suivi de Note sur la structure des paradigmes; suivi de Sur la dualité de la poétique. Liège : PUL. 
correspond à l'opération du « mélange », qui vise à défaire un «tri » antérieur, et qui implique la diminution de l'intensité et de la tension. Soit le schéma tensif (Figure 1) :

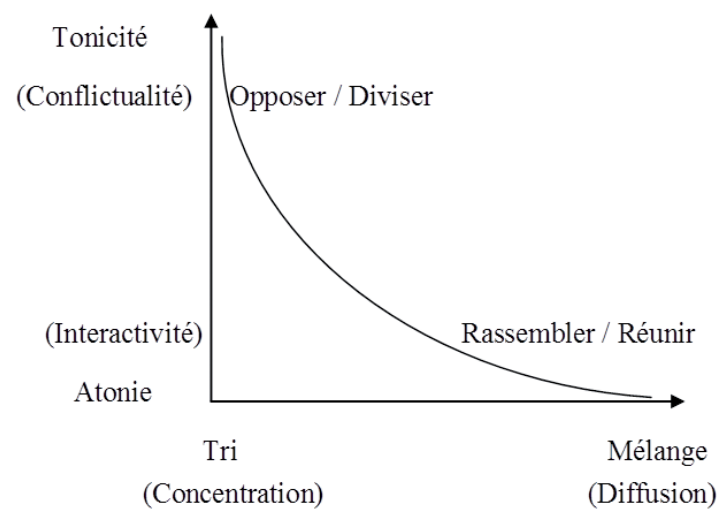

Figure 1 : Schéma tensif

Ce schéma, loin d'être un modèle paradigmatique figé, met en avant une syntagmatique : entre les deux positions définies qui servent de points de repère, il existe non seulement un nombre infini de positions intermédiaires, mais aussi d'innombrables mouvements et transformations possibles d'une position à une autre. Plus on rassemble, moins on divise, et inversement : une concurrence continuelle s'instaure entre les diverses instances discursives et sociales pour occuper une position sur le schéma, position qui restera pourtant instable, à tout moment menacée par les forces de l'un des deux pôles, sinon par celles des deux ${ }^{1}$.

Après cette analyse succincte au niveau de l'impersonnel de l'énonciation, entrons dans le vif de notre sujet, à savoir la manifestation de ce système de valeurs / antivaleurs, rattaché à d'autres valeurs / antivaleurs, dans le débat Hollande-Sarkozy.

1 Par ailleurs, le rassemblement et la division peuvent s'effectuer avec des intensités (tonicité et tempo) et des extensités (nombre de personnes impliquées, par exemple) variables. On obtiendra ainsi des schémas tensifs du rassemblement (et de la division). Les quatre principaux types de rassemblement (ou de division), sous cet angle, seront donc : 1 . local et intense ; 2 . massif et intense ; 3 . local et faible ; 4. massif et faible. Chaque position pourrait naturellement s'appliquer à de nombreux exemples dans la vie réelle et dans les discours effectifs. 


\section{Débat Hollande-Sarkozy}

\subsection{Rassemblement}

\subsubsection{Exclusivité d'une valeur}

Dans ce débat, comme on le verra, le rassemblement se présente comme une valeur essentielle à partir de laquelle la structure narrative se forme et autour de laquelle les autres valeurs se situent. En revanche, la revendication de cette valeur est concurrentielle : chaque candidat tente de nier sa présence chez l'autre et de la considérer comme une valeur qui lui est propre.

L'idée du rassemblement est d'abord lancée par Hollande. Selon lui, le président sortant - son rival, présent sur la scène - a divisé et opposé les Français :

Je veux être aussi le président du rassemblement. Pendant trop d'années, les Français ont été opposés systématiquement les uns par rapport aux autres, divisés, et donc je veux les réunir.

Comme on le sait, la textualisation ${ }^{1}$ joue un rôle déterminant dans la construction de l'effet de sens : celui-ci peut complètement se modifier en fonction du moment où une entité discursive s'introduit dans une énonciation. Le lancement de cette thématique par Hollande dès le début de son discours montre son importance, ce qui est d'ailleurs confirmé par la suite, car le rassemblement sert comme une sorte d'isotopie axiologique, qui apparaît et réapparaît à des moments clés, et qui est présupposé ou impliqué par d'autres affirmations.

Ici, en employant les termes " trop » et " systématiquement », Hollande souligne la gravité et l'excès de la situation actuelle : un seuil est dépassé et une nécessité de changement s'impose. Il définit en réalité deux territoires, celui du rassemblement et de la réunion qu'il défend, et celui de la division et de l'opposition qu'il attribue à Sarkozy, et qu'il condamne.

1 La textualisation désigne la "[d]isposition des données discursives selon les contraintes dues à la linéarité de la manifestation verbale. Ainsi, deux actions parallèles ne peuvent être narrées simultanément ». Bertrand, D. (2000). Précis de sémiotique littéraire (p. 266). Paris : Nathan. 
Sarkozy, à son tour, tout en reconnaissant cette dichotomie, n'accepte point d'appartenir au territoire de la division et de l'opposition, mais à celui du rassemblement. Lui aussi, exactement comme son rival, essaie d'exclure l'autre de ce territoire :

Le rassemblement, c'est un très beau mot, c'est une très belle idée. Mais il faut y mettre des faits. Le rassemblement, c'est quand on parle au peuple de France, à tous les Français. Je ne suis pas l'homme d'un parti. Je ne parle pas à la gauche. Hier, je me suis adressé à tous les Français, pas simplement aux syndicalistes, qui ont parfaitement un rôle à jouer. Le rassemblement, c'est de parler à tous, y compris à ceux qui n'ont pas vos idées. Parce que lorsqu' on est président de la République, on est président bien sûr de ceux qui ont voté pour vous, mais on l'est aussi de ceux qui n'ont pas voté pour vous. C'est peut-être ce qui fait notre différence. Au peuple de France dans son ensemble.

Et Hollande réplique ainsi à cette affirmation : « Oui, c'est sans doute ce qui fait notre différence ».

On constate que Hollande et Sarkozy débutent tous deux leur tour de parole en évoquant cette valeur, ce qui fait preuve de la centralité de la question pour l'un comme pour l'autre. D'après cet extrait, ce qui est considéré comme critère pour distinguer la valeur du rassemblement de l'antivaleur de la division, c'est le choix de l'énonciataire : à qui s'adresse-t-on ? À « un parti », aux « syndicalistes », ou à « tous les Français », au « peuple de France dans son ensemble »?

Les deux candidats se rejoignent sur deux points : d'une part, le rassemblement est une valeur fondamentale ; et d'autre part, il s'agit d'un territoire exclusif, propre, duquel l'autre est exclu. D'où leur accord sur le fait que cette valeur est l'un des principaux éléments constituant leur « différence». Hollande, dans sa réplique, utilise même un adverbe qui indique un degré de certitude plus élevé par rapport à Sarkozy : « peut-être » est remplacé par « sans doute».

Dès son deuxième tour de parole, Hollande reprend cette question : « Je veux revenir sur le rassemblement, parce que je pense, comme vous, que c'est une notion essentielle pour notre pays ». De fait, le rassemblement devient un leitmotiv jusqu'à la fin du débat. 
Pour rendre cette valeur exclusive, chacun des candidats doit, d'une part, la montrer virtualisée chez l'autre et, d'autre part, la montrer réalisée ou réalisable chez lui-même. La virtualisation de cette valeur chez l'autre s'effectue par la remise en cause de son passé - stratégie fréquente dans les débats politiques. Chacun tente de montrer que l'autre ou son camp, au lieu de rassembler, a divisé. Par exemple, selon Hollande, Sarkozy a distingué « les syndicalistes qui [lui] plaisent et les syndicalistes qui ne [lui] plaisent pas », il a opposé « les salariés du privé » et « du public », il a cherché à " savoir qui est né ici, depuis combien de générations, ou qui habite là », etc. À ses yeux, Sarkozy a «nommé [ses] proches partout, dans tous les ministères, dans toutes les préfectures, y compris dans les établissements bancaires », il « a reçu, comme président de la République, les parlementaires de la majorité à l'Élysée », donc il s'est « comporté comme un chef de parti », « comme un chef de majorité », il a « eu une présidence partisane, partiale », etc.

De l'autre côté, Sarkozy suggère que Hollande ne cesse « de parler au peuple de gauche, au parti socialiste », qu'il ne cesse « d'être entouré par le parti socialiste, par Monsieur Mélenchon et par Madame Joly », que son «État-major de campagne n'est composé que de socialistes », qu'il n'aime «pas les riches [à savoir] à partir de 4000 euros », qu'il considère certains Français comme "des sous-citoyens ». Ou bien, il affirme : "Vous avez dit que tous ceux qui avaient travaillé avec la majorité sortante - puisque, pour vous, c'est déjà fait -, vous ne travailleriez pas avec eux »; " Mais c'est le même François Hollande, qui, quand il s'enflamme en mimant François Mitterrand dans les meetings dit: «Je ne garderai aucun des magistrats, aucun des policiers, aucun des préfets qui ont travaillé aux côtés de Nicolas Sarkozy»? »

Il attaque par ailleurs, plus généralement, la gauche. Selon lui, « l'esprit du rassemblement irrigue toute la société française, peut-être à l'exception d'une partie de la gauche », ce pour quoi il présente des exemples ${ }^{1}$.

1 Axel Kahn qui « compare le rassemblement du Trocadéro d'hier pour la fête du travail au congrès de Nuremberg », les « magistrats qui s'engagent pour [le] soutenir en violation de toutes les règles syndicales », le leader de la CGT qui « appelle à voter pour le candidat socialiste, en violation de toutes les traditions syndicales ». Il trouve également que la gauche l'a insulté - ce qui est l'une des manifestations de la division -, et que Hollande avec son silence a validé ces propos : «Quand on m'a comparé à Franco, à Pétain, à Laval - et pourquoi pas Hitler ? - vous n'avez pas dit un mot. [...] Madame Aubry me traite de Madoff, 183 ans de prison, et que le leader de la famille ne dit rien, c'est qu'il cautionne ». 
Plusieurs des remises en cause se concrétisent à travers la présupposition - procédé très important tout au long du débat. À titre d'exemple, Sarkozy indique : «Je ne suis pas l'homme d'un parti. Je ne parle pas à la gauche ». Il aurait pu dire : « Je ne parle pas à la droite », ce qui montrerait aussi bien son esprit de rassemblement. Mais en utilisant le terme de « gauche » à la place de celui de « droite », il fait entendre, en présupposé, une autre voix (cf. la théorie de la polyphonie chez Ducrot), celle de Hollande, candidat qui, selon le locuteur, divise la France.

À ces attaques réciproques, chacun des candidats réagit notamment de deux façons :

(i) par le reniement de l'affirmation de l'autre, tantôt avec des formules courtes comme : «Non », « Non, jamais », « Mais pour moi non plus » (formulation qui répond à un sous-entendu insinué à travers la parole de l'autre) ; tantôt avec des formules plus longues qui permettent de se défendre et de délégitimer son concurrent plus explicitement. Par exemple Sarkozy déclare : « J'ai invité tous les parlementaires », « Mais comment osez-vous dire que j'ai eu une présidence partisane, alors que, dans la majorité, on m'a reproché un nombre incalculable de fois de faire la part trop belle à ceux qui m'avaient combattu, à ceux qui n'avaient pas mes idées ?»; ou bien Hollande avance : " Tous ceux qui me suivent savent que je n'ai jamais prononcé de telles phrases », « Ce n'est pas du tout ce que j'avais dit », « Vous avez fait une erreur d'interprétation », etc. Un autre terme, plus significatif que l'" erreur », apparaît à plusieurs reprises dans le débat : " mensonge » (parfois associé à « calomnie »), qui, contrairement à l'« erreur », suppose la volonté, chez l'autre, de cacher une vérité, de tromper les interlocuteurs, ce qui est naturellement un vice moral.

(ii) en évoquant un acte similaire dans le camp de l'autre (soit l'argument dit ad hominem). Par exemple, Sarkozy signale : «Monsieur Mitterrand ne vous recevait-il pas ? », «Monsieur Mitterrand avait nommé un de ses proches "; ou bien Hollande affirme : «Vos amis, vos propres amis, m'ont comparé à je ne sais quel bestiaire. J'ai eu droit à tous les animaux des zoos »; «Moi, est-ce que je me suis plaint que la présidente du patronat français ait dit que vous aviez fait un boulot extraordinaire et que moi, mes propositions n'étaient pas bonnes ? »... 
D'autre part, chacun défend son passé et présente de nouvelles propositions. Par exemple Sarkozy donne « une preuve » de « cet esprit de rassemblement qui [1'] anime pendant cinq ans » : " C'est qu'il n'y a jamais eu de violence pendant les cinq ans de [son] quinquennat, qu'[il n'a] jamais été amené à retirer un texte qui aurait pu blesser ou créer un climat de guerre civile ». De plus, il insiste sur le fait qu'il a déjà nommé plusieurs "des personnalités de gauche » à différents postes $^{1}$. En résumé il défend ainsi son bilan : « Il y a ceux qui parlent de rassemblement et puis il y a ceux qui l'ont fait ». À son tour, Hollande souligne qu'il ne nommera "personne sans que les Commissions parlementaires en décident » pour que l'opposition soit « directement associée », qu'il renoncera " au statut pénal du chef de l'État », qu'il ne recevra " pas les parlementaires de la majorité à l'Élysée », qu'il ne participera «pas à des collectes de fonds pour [s]on propre parti, dans un hôtel parisien », etc.

Un carré sémiotique établi par Denis Bertrand, Alexandre Dézé et Jean-Louis Missika (2007, pp. 31-32), qui, selon les auteurs, présente " quatre grands régimes axiologiques », permet de structurer sous un autre jour les affirmations des deux candidats à propos du rassemblement, mais aussi à propos d'autres thèmes, ce qui sera d'ailleurs confirmé par les autres extraits que nous allons tirer du corpus au fur et à mesure (Figure 2):

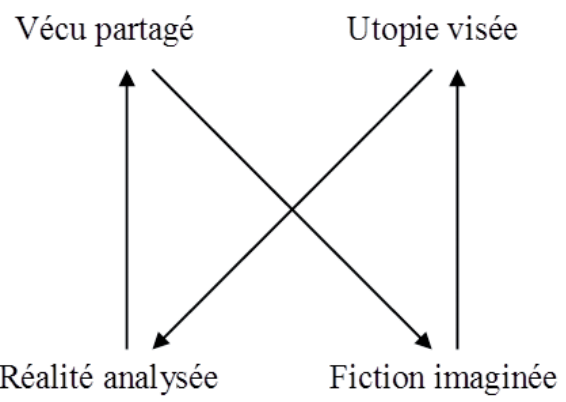

Figure 2 : Carré sémiotique

1 « À la Cour des Comptes, j'ai nommé un député socialiste [...] J'ai nommé au Conseil Constitutionnel un ancien collaborateur de Monsieur Mitterrand [...] J'ai nommé à la Présidence de la SNCF l'ancien Directeur de cabinet de Madame Aubry [...] J'ai nommé dans les gouvernements, aux côtés de Monsieur Fillon, des personnalités de gauche $» . .$. 
Toutes les positions de ce carré sémiotique sont présentes chez les candidats, mais il semble que, d'une part, la plupart des discussions se rattachent à la position de "réalité analysée ", et que, d'autre part, chez Hollande, à la fois la position de " vécu partagé " et celle d'« utopie visée » soient plus fréquentes que chez Sarkozy. Ce qui pourrait s'expliquer par le fait que le passé des deux candidats est bien différent : Sarkozy est non seulement le président sortant, mais aussi dans le gouvernement depuis des années, alors que Hollande n'a jamais été dans le gouvernement. Le débat est donc majoritairement centré sur le bilan de Sarkozy et sur les propositions de Hollande.

\subsubsection{Valeur et public}

Par ailleurs, comme on le sait, ce débat est composé d'une double énonciation : entre les deux candidats d'une part, et entre les candidats et le public de l'autre. Puisque la finalité pragmatique de ce débat, c'est de convaincre et / ou de persuader le public ${ }^{1}$, et d'obtenir plus de votes le jour des élections, cette insistance si forte sur le rassemblement révèle que celui-ci est une valeur centrale pour le public. En réalité, le public occupe la place du destinateur dans le schéma narratif proposé par Greimas, à savoir l'actant qui est, en amont, la source des valeurs, qui les définit et met en jeu; et aussi celui qui, en cours d'action, juge du degré de conformité des actes des sujets par rapport à ces valeurs ; et finalement celui qui, en aval, sanctionne. Pour obtenir une sanction positive, chaque candidat doit donc montrer qu'il incarne au mieux les valeurs définies par le destinateur. Aussi chacun essaie-t-il d'afficher qu'il veut, qu'il peut et qu'il sait rassembler, et que l'autre ne veut pas, ne peut pas et ne sait pas rassembler.

\subsection{D'autres valeurs rattachées}

Durant ce débat, d'autres valeurs sont aussi évoquées. Plusieurs d'entre elles - notamment celles mises en avant par Hollande -, relèvent du même registre axiologique que le rassemblement, celui des valeurs d'univers. Nous proposons d'examiner principalement trois d'entre elles, en essayant de creuser leur rapport avec le rassemblement : la

1 Il s'agit d'un « macro-acte » (cf. Frédéric Nef) modal, plus précisément factitif : créer un faire vouloir-faire ou, dans sa forme plus intense, un faire ne-pas-vouloir ne-pas-faire (voter pour lui) et / ou un faire ne-pas-vouloir-faire ou faire vouloir-nepas-faire (voter pour l'autre). 
justice, la confiance et l'espoir, le respect et la proximité. Chemin faisant, d'autres valeurs comme la vérité, la responsabilité, l'engagement et la stabilité seront abordées.

\subsubsection{Justice}

Hollande affirme dans sa parole liminaire :

Je serai le président de la justice, parce que nous traversons une crise grave, dure, qui frappe notamment les plus modestes, les plus travailleurs, ceux qui sont les plus exposés. Et donc je veux que la justice soit au cœur de toutes les décisions publiques. Les privilégiés ont été trop protégés. Et donc ce sera la justice fiscale, la justice sociale, la justice territoriale qui inspireront mon action. Je serai aussi le président du redressement. Ce sera un effort très long qui appellera la mobilisation de tous, de tous les acteurs. Et c'est pourquoi je veux être aussi le président du rassemblement. Pendant trop d'années, les Français ont été opposés systématiquement les uns par rapport aux autres, divisés, et donc je veux les réunir. Car je considère que c'est de toutes les forces de la France dont nous avons besoin. Et c'est ainsi que reviendra la confiance. Elle est indispensable à l'action publique - la confiance à l'égard d'un président, la confiance à l'égard d'une politique, d'une vision, d'une idée de nous-mêmes, qui nous permet de repartir de l'avant. C'est le sens du changement que je propose.

Selon ce passage, le rassemblement servira de moyen pour faire régner la justice et le redressement, ce qui entraînera la confiance. Soit le syntagme suivant :

Rassemblement $\rightarrow$ justice et redressement $\rightarrow$ confiance

Nous reviendrons sur la confiance plus loin. Pour l'instant, précisons quelques points au sujet de la justice. Comme on l'a déjà vu, si la valeur d'absolu concentre l'intensité sur une seule grandeur (ou sur une seule classe de grandeurs), la valeur d'univers implique la distribution de l'intensité sur l'ensemble des grandeurs (ou sur l'ensemble des classes 
de grandeurs). Donc, la justice est la figure par excellence de la valeur d'univers. C'est dire que la justice et le rassemblement font partie du même univers de valeur. Selon la même logique, l'injustice, comme la division et l'opposition, est une valeur d'univers (ici une antivaleur). Le même type de conflit qui existe autour de la dichotomie rassemblement / division voit le jour à plusieurs reprises chez les candidats à propos de celle de justice / injustice. Chacun cherche à ce que la justice soit une valeur exclusive, il faut donc la prouver chez soi et la nier chez l'autre ; on peut néanmoins noter que l'insistance de Hollande est ici visiblement plus intense. Par exemple, d'après lui, sous Sarkozy, « les injustices [...] ont été creusées, les inégalités [...] ont été aggravées », « la justice [...] a manqué tout au long de ces cinq dernières années »...; en revanche, il pense rétablir la justice « partout», il soutient le fait qu'elle soit « au cœur de toutes les décisions publiques », il exige même d'être jugé « sur les décisions qu' [il aura] prises en fonction de ce critère : juste ou pas». Il annonce qu'il constituera « un gouvernement qui sera paritaire », il envisage de faire « fonctionner la justice de manière indépendante $»^{1}$, etc. De son côté, Sarkozy trouve qu'entendre parler de «l'indépendance de la justice » par Hollande, "c'est une plaisanterie », car François Mitterrand " présidait le Conseil Supérieur de la Magistrature », alors que Sarkozy « a supprimé la présidence du Conseil de la Magistrature par le président de la République »...

Soulignons également que plusieurs des propositions de Hollande vont dans le sens du rassemblement et de la justice : « acte de décentralisation », considération des «partenaires sociaux », engagement de " grands débats citoyens », introduction de « la représentation proportionnelle pour les élections législatives de 2017 [pour] que l'ensemble des sensibilités politiques soit représenté ». Il s'agit donc à chaque fois de la distribution de l'intensité, de la force, de la concentration, sur l'axe de l'extensité, de la spatialité, du nombre. Ce qui implique la détente.

\subsubsection{Confiance et espoir}

Dans ce débat, la confiance apparaît comme une autre valeur articulée au rassemblement. Elle est par ailleurs directement liée à l'espoir, qui est une passion qui s'oppose à la peur. Hollande affirme :

1 Dans ce dernier exemple, il s'agit, bien entendu, de la justice en tant qu'institution, mais il va de soi que celle-ci est directement liée à la justice en tant que valeur. 
Il n'y a pas de peur à installer. Que les Français n'aient pas peur. Il n'y aura pas la spéculation que vous avez réclamée. Il n'y aura pas la comparaison avec l'Espagne. Il n'y aura pas l'invasion par des cohortes étrangères. Non, les lois de la République seront appliquées. La gestion sera saine. Les finances publiques seront rétablies, et l'économie sera activée. Voilà ce que je voulais vous dire. Moi je ne veux pas porter une peur, même pas la peur de votre propre reconduction. Ce que je souhaite, c'est que les Français reprennent confiance et espoir.

Il s'agit d'un candidat qui donne de la confiance et de l'espoir face à un autre qui suggère de la peur (au cas où c'est son rival qui gagne) ${ }^{1}$. La confiance est une composante de l'espoir : celui-ci correspond à une attente fiduciaire, une attente associée à la confiance dans un autre sujet ou dans un destinateur. Si cette attente est associée à la défiance (absence de confiance), elle engendre la peur. C'est la modalité du croire qui détermine la distinction. Pourtant, le rapport entre l'espoir et la peur n'est pas catégoriel, mais graduel, encore mieux tensif, dans la mesure où c'est le degré de la confiance dans un destinateur ou un autre sujet qui fait qu'un actant a plus ou moins d'espoir ou plus ou moins de peur : plus il a confiance, plus il a espoir et moins il a peur ; moins il a confiance, moins il a espoir et plus il a peur. Il est question de la confiance dans un destinateur, ou dans un autre sujet, pour qu'il opère pour le sujet la conjonction avec un objet de valeur (ou la disjonction d'un anti-objet de valeur).

Si Hollande tient à donner de l'espoir, c'est que, selon lui, la confiance du peuple dans le président de la République, son rival sur la scène, est affaiblie.

L'espoir correspond à la détente, contrairement à la peur qui correspond à la tension. L'espoir va donc dans le sens du rassemblement et de la justice dans la mesure où ils impliquent tous trois la détente au détriment de la tension, impliquée par la division et l'injustice.

$\mathrm{Si}$, selon le dernier extrait cité, Hollande déclare qu'il ne veut en aucun cas faire peur, même quant au choix de son rival, celui-ci installe systématiquement la peur concernant le choix du premier. Pour cela, il utilise deux procédés conjoints : a) il décrit la situation actuelle de la France et du monde comme particulièrement difficile et complexe,

1 C'est Hollande qui lexicalise la peur, passion que, selon lui, Sarkozy veut engendrer chez le public. 
d'autant plus qu'ils subissent des changements à une vitesse démesurée ; b) il remet en cause les compétences de Hollande (pouvoir-faire et savoir-faire), insuffisantes pour présider la France dans cette période historique, tout en créant un effet de contraste avec son pouvoir-faire et son savoir-faire maximaux. Par exemple, il indique :

Nous sommes dans un monde dangereux, un monde difficile où il faut savoir prendre des décisions, tenir un cap et assumer, assumer sa responsabilité. J'ai beaucoup réfléchi avant d'être candidat. Si je le suis, c'est parce que j'ai la passion de la France et que je souhaite vous conduire dans ce monde difficile pour les cinq années qui viennent.

Je crois que le président de la République est quelqu'un qui assume ses responsabilités, qui doit être profondément engagé, qui n'a pas le droit de dire qu'il ne peut pas, qu'il ne veut pas, qu'il aurait voulu mais qu'il n'a pas pu, qui doit être en première ligne, qui doit conduire un certain nombre de changements extraordinairement difficiles dans un monde lui-même extraordinairement complexe. [...] Je pense qu'un président de la République ne peut pas dire, comme le disait, devant le désastre de Vilvorde, Monsieur Jospin : "On n'y peut rien ». Je pense qu'un président de la République ne peut pas dire, comme le disait François Mitterrand dans une émission restée célèbre, sur le chômage : "On a tout essayé, on n'y peut rien ». Je pense qu'un président de la République, c'est quelqu'un - surtout avec le quinquennat - qui assume ses responsabilités, qui prend des décisions, qui est mûri par l'expérience des crises qu'il a eu à affronter. C'est sans doute la fonction la plus difficile qui soit, une fonction que j'ai apprise pendant cinq ans, à laquelle j'ai tout donné de mon énergie, de mon expérience, où je n'ai pas tout réussi. Cette expérience, j'ai considéré, au moment historique que vit la France, à un moment où le monde bouge à une vitesse stupéfiante - il y a un monde nouveau qui est en train d'arriver, alors que le monde ancien n'a pas encore disparu - qu'on ne pouvait pas s'en remettre aux vieilles lunes du passé. 
Sarkozy présente l'état actuel du monde de façon objectivée, en effaçant les traces de subjectivité : "Nous sommes dans un monde dangereux, un monde difficile », « un monde [...] extraordinairement complexe ", « au moment historique que vit la France, à un moment où le monde bouge à une vitesse stupéfiante "... C'est comme s'il s'agissait d'un discours débrayé par rapport au locuteur, inhérent au réel, universellement admis. C'est dans ce contexte que le locuteur prend position, en mettant l'accent sur ses compétences. En effet, il s'agit d'un raisonnement simple en trois temps : (i) le monde est difficile ; (ii) je suis merveilleusement à la hauteur (mais pas du tout l'autre) ; (iii) il faut me choisir. En ce qui concerne le premier fait, puisqu'il se trouve à la base de l'argumentation, son objectivation, en créant une illusion (impression) référentielle, servirait en quelque sorte de garant pour la suite, et favoriserait ainsi l'adhésion de l'énonciataire.

Le contraste modal qui apparaît dans le deuxième moment de l'argumentation de Sarkozy (son pouvoir-faire et son savoir-faire d'un côté ; le ne pas savoir-faire et le ne pas pouvoir-faire de son rival et du camp de celui-ci de l'autre) ne se limite pas à ces passages, mais il est présent de façon systématique tout au long de son discours, quelle que soit la thématique abordée : «vous ne saviez pas », «je vais vous apprendre quelque chose », « vous vous trompez », « votre incapacité », « vous serez incapable », « une grande incompétence », « depuis si longtemps éloigné des dossiers », «Monsieur Hollande connaît mal l'Europe », «J'ai participé à tous les sommets européens et vous n'avez participé à aucun », etc. ${ }^{1}$

Par ailleurs, dans ces deux passages, d'autres valeurs chères à Sarkozy se font jour, à savoir l'engagement et l'assomption des responsabilités : non seulement il faut avoir des compétences, mais il faut aussi être engagé et il faut assumer ses responsabilités. Le Petit Robert définit respectivement « assumer» et « engagement » ainsi : «Prendre à son compte ; se charger de »; " Action de se lier par une promesse ou une convention». Dans les deux cas, plusieurs modalités sont requises : le savoir-faire, le pouvoir-faire, le vouloir-faire, mais aussi le devoirfaire car la question éthique de la relation à autrui est en jeu. Il s'agit, en effet, de ce que Paul Ricœur appelle identité « ipse » (1990), c'est-

1 De l'autre côté, on peut voir la remise en cause des compétences de Sarkozy par Hollande, mais les cas sont beaucoup moins nombreux et portent en général sur des points plus précis et restreints : «Sur les carburants, vous dites que vous ne pouvez rien $[. .$.$] Vous dites que vous êtes impuissant et que vous ajouterez un peu plus de$ concurrence », " cette incapacité qui est la vôtre de maîtriser la dépense publique », « Vous ne savez pas faire les compromis »... 
à-dire, pour reprendre les formulations de Jacques Fontanille, d' « une construction par maintien et permanence d'une même direction ", de " l'instance que l'on reconnaîtrait à la constance et au maintien d'une visée » (2005, p. 23). L'engagement et l'assomption aussi, comme les valeurs précédentes, sont partagés : chacun des candidats les revendique et accuse l'autre de ne pas les revendiquer. Par exemple Hollande dit : « Devant cette situation, j'ai pris un engagement et je l'assume », « Je vais prendre des engagements devant les Français », "Je fais un autre engagement », "C'est pour la jeunesse que je veux m'engager pour le prochain quinquennat ", " J'assume là encore ce choix », " Je les assume parfaitement $» . .$. ; d'autre part, Sarkozy accuse Hollande de ne pas avoir assumé : « Mais assumez vos convictions », « Vous avez été incapables d'assumer une interdiction », " Ne vous défaussez pas à ce point-là », etc., et il répond ainsi à la même accusation qui lui est adressée par Hollande :

Monsieur Hollande peut me reprocher bien des choses et je le comprends bien, mais pas de ne pas assumer mes responsabilités. On m'a même reproché le contraire. J'ai toujours assumé mes responsabilités et je considère que c'est normal quand on est président de la République.

L'engagement et l'assomption font écho à la stabilité dans les positions, une autre valeur importante de ce débat : chacun des candidats tâche de la virtualiser chez l'autre et de la montrer réalisée chez lui'. En effet, un homme politique qui n'est pas engagé, qui n'assume pas, qui n'est pas stable, n'a pas respecté la relation éthique (le devoirfaire) qui le lie à autrui et n'est pas digne de confiance ; d'où la primordialité de ces valeurs.

Ainsi, selon Sarkozy, étant donné la situation cruciale ainsi que les contrastes modaux entre les deux candidats (vouloir-faire, savoir-faire,

1 Par exemple, Sarkozy dit : « vous changez plusieurs fois de position », « Si vous n'êtes pas quelqu'un qui varie comme une girouette, c'est extraordinaire »; de même Hollande affirme : «Et puis là, aujourd'hui, vous changez encore d'objectif »; «Vous avez parfaitement le droit de changer, moi je ne change pas », "Vous aviez pris cette position. Vous étiez déjà président de la République. Vous en changez, vous avez le droit », « En 2008, une nouvelle fois vous avez changé d'avis », « J'essaie d'avoir une cohérence dans les convictions. Je n'en change pas en fonction des circonstances ou des votes qui peuvent intervenir au premier tour d'une élection présidentielle. Je préfère tenir bon sur une position que je défends depuis des années plutôt que d'en changer sous la pression des circonstances », et Sarkozy répond ainsi à cette dernière affirmation : « Monsieur Hollande, je sais que vous avez le sens de l'humour mais vous, tenir bon sur vos convictions, pas vous et pas ça ». 
pouvoir-faire, devoir-faire), le choix des Français est décisif : il s'agira d'un événement au sens le plus fort du terme ${ }^{1}$. D'où l'importance cruciale de ce débat :

Je veux que ce soir, ce soit un moment d'authenticité où chacun donne sa vérité et que les Français en liberté choisissent - pas avec des formules creuses, pas avec des propos entendus où chacun se dénierait les qualités qu'on pourrait se prêter par ailleurs. En vérité. C'est un choix historique. La France n'a pas le droit à l'erreur. Nous ne sommes pas dans une crise, Monsieur Hollande, mais dans des crises. Et mon ambition, ce serait qu'à la fin de ces deux heures, tous ceux qui hésitent, tous ceux qui réfléchissent se disent au moins, quel que soit mon choix, j'ai une idée précise des éléments qui vont me permettre de le faire².

Il est également à noter que la réclamation de la vérité est un thème qu'évoque Sarkozy à maintes reprises au cours du débat : il déclare qu'il tient à ce que le paraître coïncide avec l'être, qu'il n'y ait pas de paraître non conforme à l'être; en d'autres termes, il affirme qu'il évite l'illusion et le mensonge (cf. carré véridictoire de Greimas). Ce qui est pourtant une évidence, car tout public n'attend que cela de la part des candidats. En réalité, Sarkozy construit un métadiscours qui aurait des effets de sens et des retombées dont les principaux seraient : (i) il met en relief un thème qui rime avec ce moment si crucial, ce choix si majeur : la vérité et l'authenticité servent de toile de fond sans laquelle toute argumentation, tout raisonnement seraient totalement impertinents ; (ii) il construit un ethos positif de lui-même : celui d'un homme sincère d'un côté, et celui d'un homme lucide de l'autre ; (iii) il construit un ethos négatif de son concurrent : il insinue que ce dernier risque de se tromper, de vouloir créer des illusions (donner des promesses vaines) ou de vouloir mentir (dissimuler ou dénaturer la réalité), ce qui est intensifié par une répétition très marquée des accusations de mensonge et de dissimulation directes : "C'est faux, c'est une contrevérité »,

1 Cf., par exemple, le concept de l' « événement » dans l'hypothèse tensive.

2 Il y a d'autres propos semblables chez lui : «Ce débat doit être ce moment de démocratie où les Français en refermant leur télévision se disent on peut aimer l'un, on peut préférer l'autre, on peut être en désaccord avec ce qu'ils disent, mais ils ne nous ont pas menti, ils n'ont pas joué l'esquive, ils ont été vrais. C'est cela notre défi, Monsieur Hollande, être vrais », " Ce débat est tellement important qu'il ne souffre aucun mensonge et aucune imprécision $\gg . .$. 
« Faux, archi-faux », « Vous voulez faire croire quoi ? », « Toujours l'ambiguïté », « Ne noyez pas le poisson », « Il y a suffisamment de choses que j'ai réussies ou pas réussies pour qu'on n'ait pas besoin d'ajouter l'outrance et le mensonge », " La vérité est là, elle vous gêne »; ou des formules plus crues : « Dans votre volonté de démontrer l'indémontrable, vous mentez », « Vous mentez de façon éhontée »... Le locuteur cherche sans doute à créer ainsi un effet persuasif, à savoir l'obtention de la confiance du public en manipulant la modalité du croire chez lui : croyez-moi, ne croyez pas l'autre !

Cette valeur tant soulignée chez Sarkozy, comme toutes les valeurs déjà examinées, est en même temps revendiquée par Hollande :

Oui, chacun aura sa vérité et chacun sera authentique. Je n'imagine pas que vous feindrez et vous ne pouvez pas penser que j'esquiverai. Donc nous parlerons face à face, directement. Nous nous dirons les choses, sans qu'il y ait quelque chose à cacher, à dissimuler.

Et lui aussi, à son tour, suggère l'idée que son rival risque de cacher quelque chose ; il dit plus d'une fois : «Dites la vérité », « $\mathrm{Ne}$ laissez pas penser que $[\ldots]$ », "C'est vous qui noyez le poisson », etc. Et il montre son mécontentement d'être accusé de menteur : « Mais vous avez toujours - c'est quand même terrible - à l'esprit le mot «mensonge», comme si c'était quelque chose que vous ressentiez très particulièrement. Vous venez encore de le répéter "; "C'est un leitmotiv qui devrait pour moi être insupportable mais qui, dans votre bouche, finit par être une habitude "; "Calomnies, mensonges, ce sont sans doute dans votre vocabulaire des compliments que je prends comme tels $\gg . .$.

Dans les accusations réciproques, deux formules sont plus fréquentes, qui confirment d'ailleurs les analyses déjà présentées : Hollande répète neuf fois : « Ce n'est pas vrai »; Sarkozy réitère à huit reprises : "C'est un mensonge ». Ces formules sont significatives dans la mesure où elles montrent que Sarkozy utilise un style tonique, alors que Hollande opte pour un style atone ${ }^{1}$, une opposition discursive presque partout valable tout au long de ce débat (tension vs détente).

1 Il s'agit de deux positions différentes sur un carré sémiotique dont les contraires sont: être vrai et être mensonge; et les subcontraires : ne pas être mensonge et ne pas être vrai. 


\subsubsection{Respect et proximité}

On remarque aussi la présence d'autres valeurs rattachées au rassemblement. Hollande, en réponse à la question de l'animatrice, Laurence Ferrari, qui lui demande : "Quel président comptez-vous être? », affirme :

Un président qui, d'abord, respecte les Français, qui les considère. Un président qui ne veut pas être président de tout, chef de tout et, en définitive, responsable de rien. [...] J'aurai toujours le souci de la proximité avec les Français. J'avais évoqué une présidence normale. Rien n'est normal quand on est président de la République puisque les conditions sont exceptionnelles. Le monde traverse une crise majeure, en tout cas l'Europe. [...] Bien sûr que le président doit être à la hauteur de ces sujets-là mais il doit aussi être proche du peuple, être capable de le comprendre.

Le discours construit par Hollande met en relief des relations intersubjectives : proximité, respect et compréhension, qui se rattachent toutes, encore une fois chez Hollande, à la détente, car dans tous les trois cas selon ce contexte, il s'agit de l'annulation de la tonicité concentrée et de la sollicitation de l'atonie diffuse. Il semble que dans le rapport entre un homme politique et le peuple, la proximité soit indispensable pour la compréhension, et qu'elle soit surtout une valeur inhérente au respect. Pour pouvoir rassembler et installer la justice, pour créer la confiance, il est important d'être proche, d'essayer de comprendre, de respecter ${ }^{1}$. Pour Hollande, ces qualités sont celles d'un président «normal », c'està-dire un président qui tendrait à impersonnaliser l'énonciation des pratiques impliquées, qui adoucirait les marques de la singularité et de la subjectivité ; ce qui correspond donc, là aussi, à la détente.

L'évocation de ces thèmes par Hollande suppose que, selon lui, son rival n'a pas été proche, compréhensif, respectueux, normal. C'est pourquoi Sarkozy reprend la question :

1 Il est à signaler que pour cette valeur aussi une certaine concurrence apparaît. Sarkozy, d'une part, se montre respectueux : « je vous respecte, je vous considère »; et d'autre part, accuse son rival de manque de respect : « Vous m'avez dit «Au revoir» hier. J'ai trouvé que ce n'était pas très respectueux pour les Français ». 
Monsieur Hollande, vous avez parlé - sans doute pour être désagréable à mon endroit - d'un président normal. Je vais vous dire : la fonction de président de la République n'est pas une fonction normale. La situation que nous connaissons n'est pas une situation normale. Votre normalité n'est pas à la hauteur des enjeux.

On observe le retour de Sarkozy sur les deux procédés qu'il utilise pour faire peur au public : complexité de la situation et manque de compétence de Hollande. C'est dire que si Sarkozy considère l'opposition principale en termes modaux (savoir-faire vs ne pas savoir-faire; pouvoir-faire vs ne pas pouvoir-faire; devoir-faire vs ne pas devoir-faire), Hollande la voit en termes de sémiotique tensive : tension vs détente ${ }^{1}$.

Par ailleurs, comme nous l'avons constaté dans la première citation de Hollande, celui-ci met en avant la notion de responsabilité, sur laquelle insiste particulièrement Sarkozy. Hollande accuse celui-ci d'avoir été " président de tout, chef de tout », mais " responsable de rien ». Rappelons que la question de la responsabilité est liée à celle de l'éthique, à savoir l'un des domaines par excellence de la construction et du développement des valeurs ${ }^{2}$. Cette corrélation entre l'assomption des responsabilités d'un côté, et le pouvoir de l'autre, peut se représenter sur un schéma (Figure 3) :

$$
\text { Assomption }\left[\begin{array}{cc}
\text { A) Président de rien, } & \text { B) Président de tout, } \\
\text { Responsable de tout } & \text { Responsable de tout } \\
\text { C) Président de rien, } & \text { D) Président de tout, } \\
\text { Responsable de rien } & \text { Responsable de rien }
\end{array}\right.
$$

Figure 3 : Schéma de la corrélation entre l'assomption des responsabilités et le pouvoir

1 Bien sûr, ici aussi, on peut en faire une interprétation modale, mais dans ce cas-là, les modalités ne porteront pas sur le même « faire» : un candidat qui ne veut pas / ne sait pas / ne peut pas détendre est confronté à un autre qui veut / sait / peut détendre.

2 Cf. à ce sujet: Fontanille, J. (2008). Pratiques sémiotiques (pp. 235-292). Paris : PUF. 
Les positions $\mathrm{B}$ et $\mathrm{C}$, pour reprendre les termes de la sémiotique tensive, sont de l'ordre de l'implicatif (X donc $\mathrm{Y}$ ), tandis que les positions $\mathrm{A}$ et $\mathrm{D}$ sont de l'ordre du concessif (X pourtant $\mathrm{Y}$ ). Évidemment, les positions indiquées sont des positions radicales, entre lesquelles se trouvent d'innombrables positions intermédiaires. La position que s'attribue Sarkozy est proche de B, mais Hollande lui attribue la position $\mathrm{D}$, à savoir la position la plus immorale (dans ce cas, le sujet n'a pas respecté un contrat fiduciaire - explicite ou implicite antérieur) ${ }^{1}$.

Quant à la position qu' attribue Sarkozy à Hollande, elle est proche de $\mathrm{C}$, car il a affirmé à plusieurs reprises que celui-ci est dépourvu de la puissance nécessaire et incapable d'assumer. Mais quelle est la position que s'attribue Hollande ? Il semble, encore une fois, que Hollande cherche une position détendue : en mettant de côté les extrémités « tout » et «rien », sa position se situerait plutôt au milieu de ce schéma, ce qui correspondrait à une formule de genre " président de quelque chose, responsable de quelque chose ». Cela fait écho à la figure du " président normal ».

\section{Pour conclure}

Dans cet article, nous avons visé à examiner la mise en discours d'un système de valeurs / antivaleurs qui se construit dans le débat Hollande-Sarkozy, en passant par une brève analyse au niveau de l'impersonnel de l'énonciation. D'une part, nous avons essayé de montrer que la dichotomie rassemblement / division constitue l'une des principales entités structurant ce débat, et que chacun des candidats

1 Hollande essaie, à maintes autres reprises, de montrer que Sarkozy a eu beaucoup de responsabilité, mieux, qu'il a été le seul responsable («président de tout»), et qu'il a échoué, mais qu'il n'assume pas (« responsable de rien ») : " je vous ai mis devant ce qui a été votre responsabilité de président », "Vous avez une très grave responsabilité », «Avec vous, c'est très simple : ce n'est jamais votre faute. Vous avez toujours un bouc émissaire. Ce n'est jamais de votre faute », "Vous êtes toujours content de vous, ce qui est d'ailleurs extraordinaire. Quoi qu'il arrive, quoi qu'il se passe, vous êtes content », "Voilà la responsabilité d'une équipe sortante depuis dix ans : la dette », "Le bilan de Nicolas Sarkozy a été décrit comme étant particulièrement lourd », etc. D'autre part, si l'ensemble du discours de Sarkozy laisse penser à une position proche de $\mathrm{B}$, il n'assume pas de façon explicite être «président de tout », car cela nuirait à son image, et impliquerait qu'il serait « responsable de tout », y compris de tous les problèmes ; c'est pourquoi lorsqu'il est obligé de se situer sur ce schéma, pour réagir contre la position que lui attribue Hollande, il n'adopte qu'une position nuancée : «Je n'ai pas évidemment le seul mérite, mais je ne peux pas être le seul coupable non plus ». 
cherche son identité politique dans le rassemblement et accuse l'autre d'être diviseur ; d'autre part, nous avons mis l'accent sur les rapports entre le rassemblement et d'autres valeurs comme la justice, l'espoir, la confiance, le respect, la compréhension, la proximité. Ces valeurs, tout en ayant des liens sémantiques et syntaxiques plus ou moins différents avec le rassemblement, ont du moins ceci en commun qu'elles correspondent toutes aux valeurs d'univers et qu'elles impliquent la détente. En effet, si le discours de Sarkozy laisse entendre qu'il faut évaluer le contraste entre les candidats en termes modaux (savoir-faire, pouvoir-faire et devoir-faire qui le caractérisent et ne pas savoir-faire, ne pas pouvoir-faire, ne pas devoir-faire qui caractérisent son rival), le discours de Hollande suggère que le contraste est à observer sur une base tensive (il aspire à la détente, alors que son concurrent s'attache à la tension). De plus, nous avons constaté que des valeurs comme responsabilité, engagement, stabilité et vérité - elles aussi revendiquées par les deux candidats - jouent un rôle déterminant dans ce débat.

Cette brève recherche peut évidemment aller bien plus loin. Par exemple, on peut se demander : quel rôle précis ce système de valeurs joue-t-il dans la trame narrative de ce débat ? Comment se rapporte-t-il à d'autres enjeux discursifs impliqués tels que le mode d'énonciation (position de l'instance du discours, présupposition, sous-entendu...), les passions et émotions, etc. ? Comment situer ce système de valeurs / antivaleurs tel qu'il se manifeste dans ce débat dans l'ensemble des discours de chacun de ces deux hommes politiques ? Comment le situer dans l'ensemble des discours politiques, aussi bien au niveau national qu'au niveau interculturel ? Les différentes cultures exploitentelles ce système de la même façon ? Existe-t-il des spécificités de ce système en fonction de chaque type de discours? Quelles sont les autres valeurs impliquées dans chaque cas?

\section{Références}

Bertrand, D. (2000). Précis de sémiotique littéraire. Paris : Nathan.

Bertrand, D., Dézé, A., \& Missika, J.-L. (2007). Parler pour gagner : sémiotique des discours de la campagne présidentielle de 2007. Paris : Presses de Sciences Po (P.F.N.S.P.).

Charaudeau, P. (2005). Le discours politique. Paris : Vuibert.

Charaudeau, P. (2013). La conquête du pouvoir : opinion, persuasion, valeur. Les discours d'une nouvelle donne politique. Paris : L'Harmattan. 
Dacheux, E. (2011). La communication. Paris : CNRS.

Ducrot, O. (1984). Le dire et le dit. Paris : Minuit.

Fontanille, J. (2003). Sémiotique du discours. Limoges : PULIM.

Fontanille, J. (2005). Soma et séma : figures du corps. Paris : Maisonneuve \& Larose.

Fontanille, J. (2008). Pratiques sémiotiques. Paris : PUF.

Fontanille, J. (2010). Une corde pour tout échange : l'invention de la valeur en interaction. Signata, 1, 311-322.

Fontanille, J., \& Zilberberg, C. (1998). Tension et signification. Liège : Mardaga.

Goffman, E. (1974). Les rites d'interaction. Paris : Minuit.

Greimas, A.J. (1983). Du sens II : essais sémiotiques. Paris : Seuil.

Greimas, A.J. (1986). De la nostalgie, Étude de sémantique lexicale. Actes sémiotiquesbulletin, 39, 5-11. Paris : EHESS-CNRS. Dans A. Hénault (Dir.) (2002). Questions de sémiotique (pp. 593-600). Paris : PUF.

Greimas, A.J., \& Courtés, J. (1979). Sémiotique : dictionnaire raisonné de la théorie du langage, t. 1. Paris : Hachette.

Guerrini J.-C., \& Majcherczak, E. (1999). L'argumentation au pluriel : polyphonie, valeurs, points de vue. Lyon: PUL.

Kerbrat-Orecchioni, C. (2005). Le discours en interaction. Paris : Armand Colin.

Kerbrat-Orecchioni, C. (2013). Corpus médiatique et interprétation : le cas des débats présidentiels. Dacoromania, 18(1), 19-34.

Landowski, E. (1989). La société réfléchie. Paris : Seuil.

Landowski, E. (1997). Présences de l'autre. Paris : PUF.

Mayaffre, D. (2012). Nicolas Sarkozy : mesure et démesure du discours (2007-2012). Paris : Presses de Sciences Po (P.F.N.S.P.).

Nef, F. (1980). Note pour une pragmatique textuelle. Macro-actes indirects et dérivation rétroactive. Communications, 32, 183-189.

Ricœur, P. (1990). Soi-même comme un autre. Paris : Seuil.

Salavastru, C. (2004). Rhétorique et politique : le pouvoir du discours et le discours du pouvoir. Paris : L'Harmattan.

Zilberberg, C. (1999). Sémiotique de la douceur. Tópicos del Seminario, Benemérita Universidad Autónoma de Puebla, 2, 31-64. Disponible sur : http://www. claudezilberberg.net/download/downset.htm.

Zilberberg, C. (2001). De l'affect à la valeur. Dans M. Castellana (Dir.), Texte et valeur (pp. 43-78). Paris : L'Harmattan.

Zilberberg, C. (2006). Éléments de grammaire tensive. Limoges : PULIM.

Zilberberg, C. (2008). Pour saluer l'événement. Nouveaux Actes Sémiotiques. Disponible sur : http://revues.unilim.fr/nas/document.php?id=2485.

Zilberberg, C. (2011). Des formes de vie aux valeurs. Paris : PUF.

Zilberberg, C. (2012). La Structure tensive suivi de Note sur la structure des paradigmes; suivi de Sur la dualité de la poétique. Liège : PUL. 